\title{
ON THE STABILITY OF GENERALIZED GAMMA FUNCTIONAL EQUATION
}

\author{
GWANG HUI KIM
}

(Received 1 July 1999)

\begin{abstract}
We obtain the Hyers-Ulam stability and modified Hyers-Ulam stability for the equations of the form $g(x+p)=\varphi(x) g(x)$ in the following settings: $|g(x+p)-\varphi(x) g(x)|$ $\leq \delta,|g(x+p)-\varphi(x) g(x)| \leq \phi(x),|(g(x+p) / \varphi(x) g(x))-1| \leq \psi(x)$. As a consequence we obtain the stability theorems for the gamma functional equation.
\end{abstract}

Keywords and phrases. Functional equations, stability of functional equations, Hyers-Ulam stability.

2000 Mathematics Subject Classification. Primary 39B22, 39B72, 39B82.

1. Introduction. In 1940, Ulam [7] raised the following problem: under what conditions does there exist an additive mapping near an approximately additive mapping? In 1941, this problem was solved by Hyers [2]. Therefore we usually say that the equation $E_{1}(h)=E_{2}(h)$ has the Hyers-Ulam stability if, for an approximate solution $f$ such as $\left|E_{1}(f)-E_{2}(f)\right| \leq \delta$, there exist a function $g$ such that $E_{1}(g)=E_{2}(g)$ and $|f(x)-g(x)| \leq \epsilon$. This stability problem has been further generalized [1, 6]. In this paper, we say that the equation $E_{1}(h)=E_{2}(h)$ has a modified Hyers-Ulam-Rassias stability if for an approximate solution $f$ of the following types.

In the sense of Rassias, for a fixed function $\psi$ such as

$$
\left|E_{1}(f)-E_{2}(f)\right| \leq \psi
$$

there exists a function $g$ such that $E_{1}(g)=E_{2}(g)$ and $|g(x)-f(x)| \leq \Phi(x)$ for some fixed function $\Phi$.

In the sense of Ger and $\breve{S} e m r l$, for a fixed function $\psi$ such as

$$
\left|\frac{E_{1}(f)}{E_{2}(f)}-1\right| \leq \psi
$$

there exists a function $g$ such that $E_{1}(g)=E_{2}(g)$ and $\alpha \leq f / g \leq \beta$ for some fixed functions $\alpha$ and $\beta$.

The aim of this paper is to give three stability theorems for the equation

$$
g(x+p)=\varphi(x) g(x) .
$$

The gamma functional equation is an example of (1.3), that is, our stability theorems are general cases of stability theorems for the gamma functional equation. Throughout this paper, let $\delta, p>0$ be fixed and $n_{0}$ be a given nonnegative integer. 
2. The Hyers-Ulam stability of $g(x+p)=\varphi(x) g(x)$. In the following theorem, we investigate the Hyers-Ulam stability for equations of the form (1.3).

THEOREM 2.1. If a function $g:(0, \infty) \rightarrow \mathbb{R}$ satisfies the following inequality:

$$
|g(x+p)-\varphi(x) g(x)| \leq \delta \quad \forall x>n_{0}
$$

and some function $\varphi:(0, \infty) \rightarrow(0, \infty)$ such that

$$
\gamma(x):=\sum_{j=0}^{\infty} \prod_{i=0}^{j} \frac{1}{\varphi(x+p i)}
$$

is bounded for all $x>n_{0}$, then there exist a unique solution $f:(0, \infty) \rightarrow \mathbb{R}$ of (1.3) with

$$
|g(x)-f(x)| \leq \gamma(x) \delta \quad \forall x>n_{0} .
$$

PRoof. For any $x>0$ and for every positive integer $n$ we define

$$
P_{n}(x)=g(x+p n) \prod_{i=0}^{n-1} \frac{1}{\varphi(x+p i)}
$$

By (2.1) we have

$$
\begin{aligned}
\left|P_{n+1}(x)-P_{n}(x)\right| & =\mid g(x+p(n+1))-\varphi(x+p n) g(x+p n) \prod_{i=0}^{n} \frac{1}{\varphi(x+p i)} \\
& \leq \delta \prod_{i=0}^{n} \frac{1}{\varphi(x+p i)} \quad \text { for } x>n_{0} .
\end{aligned}
$$

Now we use induction on $n$ to prove

$$
\left|P_{n}(x)-g(x)\right| \leq \delta \sum_{j=0}^{n-1} \prod_{i=0}^{j} \frac{1}{\varphi(x+p i)}
$$

for all $x>n_{0}$ and for all positive integers $n$. For the case $n=1$ the inequality (2.6) is an immediate consequence of (2.1). Assume that (2.6) holds true for some $n$. It then follows from (2.5) and (2.6) that

$$
\left|P_{n+1}(x)-g(x)\right| \leq\left|P_{n+1}(x)-P_{n}(x)\right|+\left|P_{n}(x)-g(x)\right| \leq \delta \sum_{j=0}^{n} \prod_{i=0}^{j} \frac{1}{\varphi(x+p i)}
$$

which completes the proof of (2.6). We claim that $\left\{P_{n}(x)\right\}$ is a Cauchy sequence. Indeed, for $n \geq m$ and $x>n_{0}$ we have 


$$
\begin{aligned}
\left|P_{n}(x)-P_{m}(x)\right| & \leq \sum_{j=m}^{n-1}\left|P_{j+1}(x)-P_{j}(x)\right| \\
& \leq \delta \sum_{j=m}^{n-1} \prod_{i=0}^{j} \frac{1}{\varphi(x+p i)} \longrightarrow 0 \text { as } m \longrightarrow \infty .
\end{aligned}
$$

Hence we can define a function $f_{0}:(0, \infty) \rightarrow \mathbb{R}$ by

$$
f_{0}(x)=\lim _{n \rightarrow \infty} P_{n}(x) .
$$

Since $P_{n}(x+p)=\varphi(x) P_{n+1}(x)$, we have

$$
f_{0}(x+p)=\varphi(x) f_{0}(x) \quad \forall x>n_{0} .
$$

We also have

$$
\begin{aligned}
\left|f_{0}(x)-g(x)\right| & =\lim _{n \rightarrow \infty}\left|P_{n}(x)-g(x)\right| \leq \delta \sum_{j=0}^{\infty} \prod_{i=0}^{j} \frac{1}{\varphi(x+p i)} \\
& =\gamma(x) \delta \quad \forall x>n_{0}
\end{aligned}
$$

which completes the proof of (2.3). If $h:\left(n_{0}, \infty\right) \rightarrow \mathbb{R}$ is an another function which satisfies (2.3) and (2.10), then it follows from (2.3) and (2.10) that

$$
\begin{aligned}
\left|f_{0}(x)-h(x)\right| & =\prod_{i=0}^{n-1} \frac{1}{\varphi(x+p i)}\left|f_{0}(x+p n)-h(x+p n)\right| \\
& \leq 2 \delta \gamma(x+p n) \prod_{i=0}^{n-1} \frac{1}{\varphi(x+p i)}
\end{aligned}
$$

for all $x>n_{0}$ and all positive integers $n$. This implies the uniqueness of $f_{0}$.

Now we extend the function $f_{0}$ to $(0, \infty)$. We define

$$
f(x):=\frac{f_{0}(x+k p)}{\prod_{n=0}^{k-1} \varphi(x+n p)} \text { for } 0<x \leq n_{0},
$$

where $k$ is the smallest natural number satisfying the inequality $x+k p>n_{0}$.

Then $f(x+p)=\varphi(x) f(x)$ for all $x>0$ and $f(x)=f_{0}(x)$ for all $x>n_{0}$. Also the following inequality holds:

$$
|f(x)-g(x)|<\gamma(x) \delta \quad \forall x>n_{0} .
$$

3. The modified Hyers-Ulma-Rassias stability of $g(x+p)=\varphi(x) g(x)$. In this section, we investigate the modified Hyers-Ulam-Rassias stability for equations of the form (1.3) in two types. The former (Theorem 3.1) is the sense of Rassias, the latter (Theorem 3.2) is the sense of Ger and Šemrl [1].

Let a mapping $\varphi$ and $\phi:(0, \infty) \rightarrow(0, \infty)$ satisfy the inequality

$$
\Phi(x)=\sum_{j=0}^{\infty} \phi(x+p j) \prod_{i=0}^{j} \frac{1}{\varphi(x+p i)}<\infty \quad \forall x \in(0, \infty) .
$$

By using an idea from paper [6] of Rassias, we can prove the following theorem. 
THEOREM 3.1. If a function $g:(0, \infty) \rightarrow \mathbb{R}$ satisfies the following inequality

$$
|g(x+p)-\varphi(x) g(x)| \leq \phi(x) \quad \forall x>n_{0},
$$

then there exists a unique solution $f:(0, \infty) \rightarrow \mathbb{R}$ of (1.3) with

$$
|g(x)-f(x)| \leq \Phi(x) \quad \forall x>n_{0} .
$$

Proof. Let $P_{n}(x)$ be defined as in the proof of Theorem 2.1. By (3.2), we have

$$
\begin{aligned}
\left|P_{n+1}(x)-P_{n}(x)\right| & =|g(x+p(n+1))-\varphi(x+p n) g(x+p n)| \prod_{i=0}^{n} \frac{1}{\varphi(x+p i)} \\
& \leq \phi(x+p n) \prod_{i=0}^{n} \frac{1}{\varphi(x+p i)} \quad \text { for } x>n_{0} .
\end{aligned}
$$

Now we use induction on $n$ to prove

$$
\left|P_{n}(x)-g(x)\right| \leq \sum_{j=0}^{n-1} \phi(x+p j) \prod_{i=0}^{j} \frac{1}{\varphi(x+p i)}
$$

for the fixed $x>n_{0}$ and for all positive integers $n$. For the case $n=1$, the inequality (3.5) is an immediate consequence of (3.2). Assume that (3.5) holds true for some $n$. It then follows from (3.4) and (3.5)

$$
\begin{aligned}
\left|P_{n+1}(x)-g(x)\right| & \leq\left|P_{n+1}(x)-P_{n}(x)\right|+\left|P_{n}(x)-g(x)\right| \\
& \leq \sum_{j=0}^{n} \phi(x+p j) \prod_{i=0}^{j} \frac{1}{\varphi(x+p i)}
\end{aligned}
$$

which completes the proof of (3.5). Now let $m, n$ be positive integers with $n \geq m$. Suppose $x\left(>n_{0}\right)$ is given. By (3.1), we have

$$
\begin{aligned}
\left|P_{n}(x)-P_{m}(x)\right| & \leq \sum_{j=m}^{n-1}\left|P_{j+1}(x)-P_{j}(x)\right| \\
& \leq \sum_{j=m}^{n-1} \phi(x+p j) \prod_{i=0}^{j} \frac{1}{\varphi(x+p i)} \longrightarrow 0 \quad \text { as } m \rightarrow \infty .
\end{aligned}
$$

This implies that $\left\{P_{n}(x)\right\}$ is a Cauchy sequence for $x>n_{0}$. Next proceeding of the proof is the same as that of Theorem 2.1.

THEOREM 3.2. Let $g:(0, \infty) \rightarrow(0, \infty)$ be a function that satisfies the inequality

$$
\left|\frac{g(x+p)}{\varphi(x) g(x)}-1\right| \leq \psi(x) \quad \forall x>n_{0}
$$

where $\varphi:(0, \infty) \rightarrow(0, \infty)$ is a function such that

$$
\gamma(x):=\sum_{j=0}^{\infty} \prod_{i=0}^{j} \frac{1}{\varphi(x+p i)}
$$


is bounded for all $x>n_{0}$ and $\psi:(0, \infty) \rightarrow(0,1)$ is a function such that

$$
\alpha(x):=\sum_{i=0}^{\infty} \log (1-\psi(x+p i)), \quad \beta(x):=\sum_{i=0}^{\infty} \log (1+\psi(x+p i))
$$

are bounded for all $x>n_{0}$. Then there exists a unique solution $f:(0, \infty) \rightarrow(0, \infty)$ of (1.3) with

$$
e^{\alpha(x)} \leq \frac{f(x)}{g(x)} \leq e^{\beta(x)} \quad \forall x>n_{0} .
$$

Proof. Let $P_{n}(x)$ be defined as in the proof of Theorem 2.1. For any $x>0$ and for all positive integers $m, n$ with $n>m$, it holds

$$
\begin{aligned}
\frac{P_{n}(x)}{P_{m}(x)}= & \frac{g(x+p(m+1))}{\varphi(x+p m) g(x+p m)} \cdot \frac{g(x+p(m+2))}{\varphi(x+p(m+1)) g(x+p(m+1))} \\
& \ldots \ldots \frac{g(x+p n)}{\varphi(x+p(n-1)) g(x+p(n-1))} .
\end{aligned}
$$

The following inequality is an immediate consequence of (3.8): for all $x>n_{0}$ and $i=0,1,2, \ldots$

$$
0<1-\psi(x+p i) \leq \frac{g(x+p(i+1))}{\varphi(x+p i) g(x+p i)} \leq 1+\psi(x+p i) .
$$

From (3.12) and (3.13), we get

$$
\prod_{i=m}^{n-1}(1-\psi(x+p i)) \leq \frac{P_{n}(x)}{P_{m}(x)} \leq \prod_{i=m}^{n-1}(1+\psi(x+p i))
$$

or

$$
\sum_{i=m}^{n-1} \log (1-\psi(x+p i)) \leq \log P_{n}(x)-\log P_{m}(x) \leq \sum_{i=m}^{n-1} \log (1+\psi(x+p i)) .
$$

Since this series converges by assumption, $\left\{\log P_{n}(x)\right\}$ is a Cauchy sequence for all $x>n_{0}$. Now we can define

$$
L(x):=\lim _{n \rightarrow \infty} \log P_{n}(x), \quad f(x)=e^{L(x)}=\lim _{n \rightarrow \infty} P_{n}(x) \quad \forall x>n_{0} .
$$

It is easy to see that

$$
f(x+p)=\lim _{n \rightarrow \infty} P_{n}(x+p)=\lim _{n \rightarrow \infty} \varphi(x) P_{n+1}(x)=\varphi(x) f(x) \quad \forall x>n_{0} .
$$

Since

$$
\frac{P_{n}(x)}{g(x)}=\frac{x+p}{\varphi(x) g(x)} \cdot \frac{g(x+2 p)}{\varphi(x+p) g(x+p)} \cdots \cdots \frac{g(x+p n)}{\varphi(x+p(n-1)) g(x+p(n-1))},
$$


we get

$$
\prod_{i=0}^{n-1}(1-\psi(x+p i)) \leq \frac{P_{n}(x)}{g(x)} \leq \prod_{i=0}^{n-1}(1+\psi(x+p i)) \quad \forall x>n_{0} .
$$

This implies, from (3.16), (3.19), and the definitions of $\alpha, \beta$, that

$$
e^{\alpha(x)} \leq \frac{f(x)}{g(x)} \leq e^{\beta(x)} \quad \forall x>n_{0} .
$$

Now it remains only to prove the uniqueness of $f$. Assume that $h:(0, \infty) \rightarrow(0, \infty)$ is another solution of (1.3) which satisfies (3.11). By (1.3),

$$
\frac{f(x)}{h(x)}=\frac{f(x+p n)}{h(x+p n)}=\frac{f(x+p n)}{g(x+p n)} \cdot \frac{g(x+p n b)}{h(x+p n)} \text { for any } x>0 \text { and } \forall n .
$$

Hence we have

$$
\frac{e^{\alpha(x+p n)}}{e^{\beta(x+p n)}} \leq \frac{f(x)}{h(x)} \leq \frac{e^{\beta(x+p n)}}{e^{\alpha(x+p n)}} \quad \forall n .
$$

By assumption,

$$
\alpha(x+p n)=\sum_{i=n}^{\infty} \log (1-\psi(x+p i)) \longrightarrow 0 \quad \text { as } n \longrightarrow \infty
$$

and similarly $\beta(x+p n) \rightarrow 0$ as $n \rightarrow \infty$. Hence, it is obvious that $f(x)=h(x)$.

4. Application to gamma functional equation. In this section, we apply our results to the stability of gamma functional equation. The following functional equation:

$$
g(x+1)=x g(x) \quad \forall x>0
$$

is called "the gamma functional equation". It is well known that the gamma function

$$
\Gamma(x)=\int_{0}^{\infty} e^{-t} t^{x-1} d t \quad(x>0)
$$

is a solution of the gamma functional equation (4.1). Jung [3, 4, 5] obtained the stability theorems of the gamma functional equation. We can obtain them from our results as follows:

COROLLARY 4.1. If a mapping $g:(0, \infty) \rightarrow \mathbb{R}$ satisfies the inequality

$$
|g(x+1)-x g(x)| \leq \delta \quad \forall x>n_{0},
$$

then there exist a unique solution $f:(0, \infty) \rightarrow \mathbb{R}$ of the gamma functional equation (4.1) with

$$
|g(x)-f(x)| \leq \frac{3 \delta}{x} \quad \forall x>n_{0}
$$


Proof. Apply Theorem 2.1 with $p=1$ and $\varphi(x)=x$. For any $x>0$

$$
\begin{aligned}
\sum_{j=0}^{\infty} \prod_{i=0}^{j} \frac{1}{x+i} & =\frac{1}{x}\left(1+\frac{1}{x+1}+\frac{1}{(x+1)(x+2)}+\cdots\right) \\
& \leq \frac{1}{x}\left(1+1+\frac{1}{2}+\frac{1}{2^{2}}+\cdots\right)=\frac{3}{x} .
\end{aligned}
$$

Then $\sum_{j=0}^{\infty} \prod_{i=0}^{j}(1 / \varphi(x+i))$ converges to some $\gamma(x)$ and $\gamma(x) \leq 3 / x$ for any $x>0$. Thus we complete the proof of Corollary 4.1 by Theorem 2.1.

COROLLARY 4.2. If a mapping $g:(0, \infty) \rightarrow \mathbb{R}$ satisfies the inequality

$$
|g(x+1)-x g(x)| \leq \phi(x) \quad \forall x>n_{0},
$$

then there exist a unique solution $f:(0, \infty) \rightarrow \mathbb{R}$ of the gamma functional equation (4.1) with

$$
|g(x)-f(x)| \leq \Phi(x) \quad \forall x>n_{0}
$$

Proof. Apply Theorem 3.1 and condition (3.1) with $p=1, \varphi(x)=x$.

Note 4.3. Jung's theorem [4] has the different domain from our's, but we can easily change to the same domain.

COROLlary 4.4. Let $\epsilon>0$ be given. If a mapping $g:(0, \infty) \rightarrow(0, \infty)$ satisfies the inequality

$$
\left|\frac{g(x+1)}{x g(x)}-1\right| \leq \frac{\delta}{x^{1+\epsilon}} \quad \forall x>n_{0}
$$

then there exists a unique solution $f:(0, \infty) \rightarrow(0, \infty)$ of the gamma functional equation (4.1) such that for any $x>\max \left\{n_{0}, \delta^{1 / 1+\epsilon}\right\}$

$$
e^{\alpha(x)} \leq \frac{f(x)}{g(x)} \leq e^{\beta(x)},
$$

where $\alpha(x):=\sum_{i=0}^{\infty} \log \left(1-\delta /(x+i)^{1+\epsilon}\right)$ and $\beta(x):=\sum_{i=0}^{\infty} \log \left(1+\delta /(x+i)^{1+\epsilon}\right)$.

Proof. If $x>\delta^{1 / 1+\epsilon}$, then $\sum_{i=0}^{\infty} \log \left(1-\delta /(x+i)^{1+\epsilon}\right)$ and $\sum_{i=0}^{\infty} \log \left(1+\delta /(x+i)^{1+\epsilon}\right)$ converge, respectively. Applying Theorem 3.1 with $p=1, \varphi(x)=x$ and $\psi(x)=$ $\delta / x^{1+\epsilon}$, we get the desired result.

\section{REFERENCES}

[1] R. Ger and P. Šemrl, The stability of the exponential equation, Proc. Amer. Math. Soc. 124 (1996), no. 3, 779-787. MR 96f:39027. Zbl 846.39013.

[2] D. H. Hyers, On the stability of the linear functional equation, Proc. Nat. Acad. Sci. U.S.A. 27 (1941), 222-224. MR 2,315a. Zbl 061.26403.

[3] S.-M. Jung, On a general Hyers-Ulam stability of gamma functional equation, Bull. Korean Math. Soc. 34 (1997), no. 3, 437-446. MR 99e:39094. Zbl 970.64405.

[4] - On the modified Hyers-Ulam-Rassias stability of the functional equation for gamma function, Mathematica 39(62) (1997), no. 2, 233-237. MR 99f:39037. Zbl 907.39026. 
[5] _ On the stability of gamma functional equation, Results Math. 33 (1998), no. 3-4, 306-309. MR 99f:39038. Zbl 907.39027.

[6] T. M. Rassias, On the stability of the linear mapping in Banach spaces, Proc. Amer. Math. Soc. 72 (1978), no. 2, 297-300. MR 80d:47094. Zbl 398.47040.

[7] S. M. Ulam, Problems in Modern Mathematics, Science Editions John Wiley \& Sons, Inc., New York, 1964. MR 43\#6031. Zbl 137.24201.

Kim: DePARTMENT OF MATHEMATICS, KANGNAM UNIVERSITY, SUWON, 449-702, KoREA

E-mail address: ghkim@kns. kangnam. ac. kr 


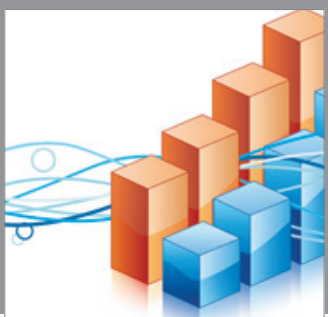

Advances in

Operations Research

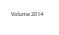

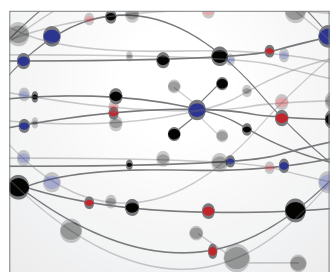

\section{The Scientific} World Journal
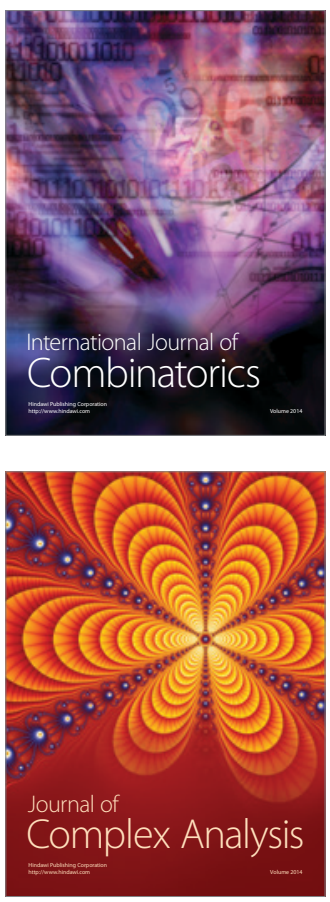

International Journal of

Mathematics and

Mathematical

Sciences
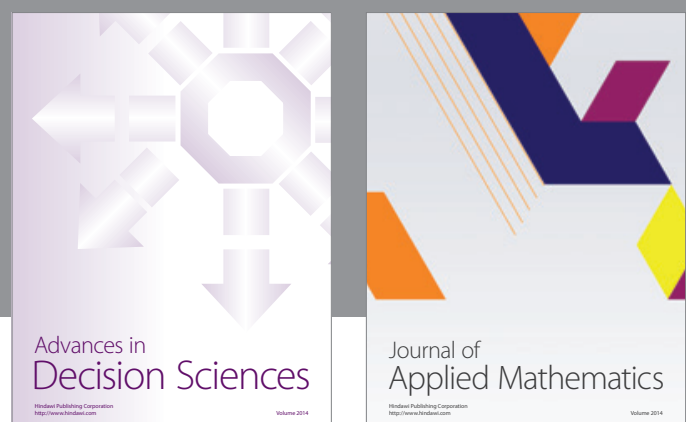

Journal of

Applied Mathematics
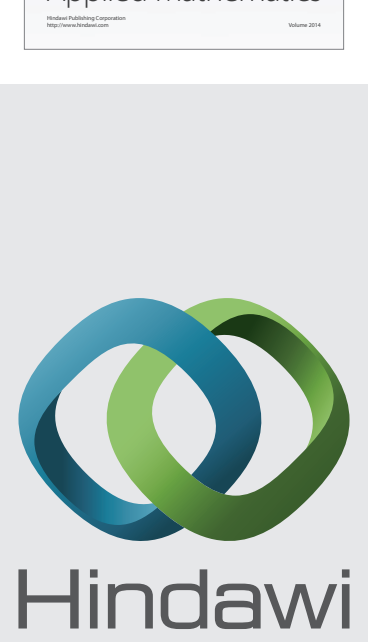

Submit your manuscripts at http://www.hindawi.com
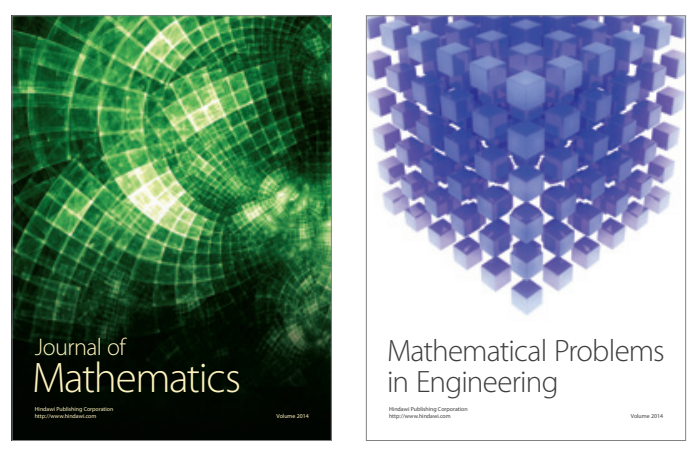

Mathematical Problems in Engineering
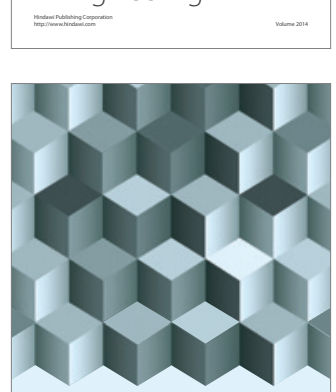

Journal of

Function Spaces
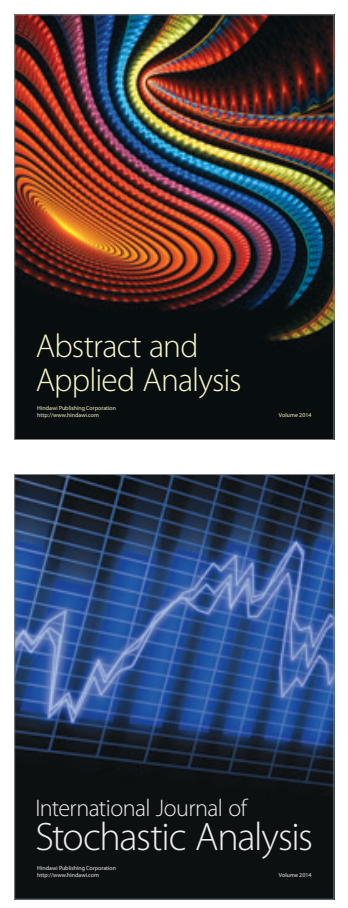

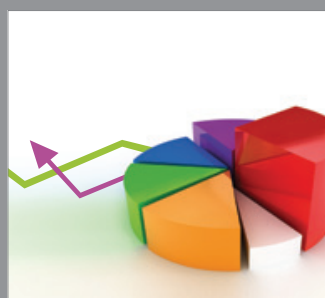

ournal of

Probability and Statistics

Promensencen
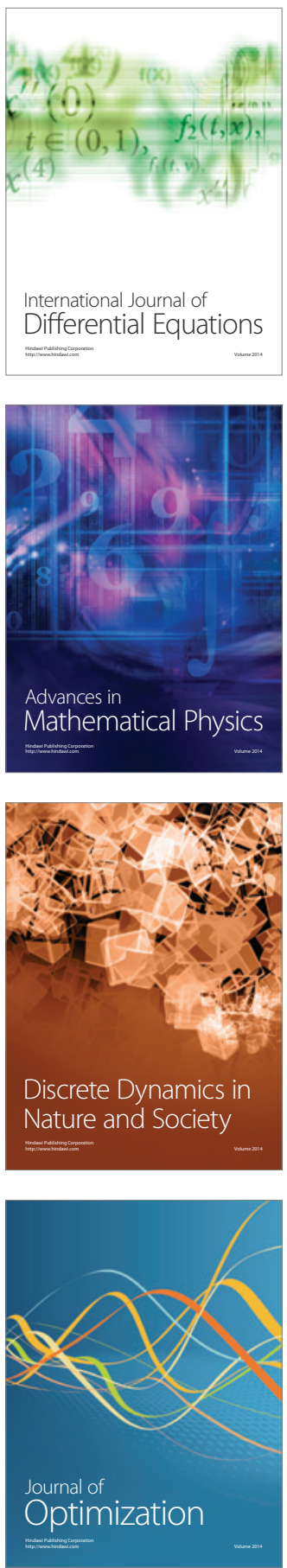